\title{
Fabric Defect Detection in Textile Manufacturing: A Survey of the State of the Art
}

\author{
Chao Li $\mathbb{D}^{1},{ }^{1}$ Jun $\mathrm{Li}^{2},{ }^{2}$ Yafei $\mathrm{Li}^{3}{ }^{3}$ Lingmin $\mathrm{He},{ }^{4}$ Xiaokang $\mathrm{Fu},{ }^{5}$ and Jingjing Chen ${ }^{1,6}$ \\ ${ }^{1}$ Zhijiang College, Zhejiang University of Technology, Hangzhou, China \\ ${ }^{2}$ Binjiang College, Nanjing University of Information Science and Technology, Nanjing, China \\ ${ }^{3}$ School of Information Engineering, Zhengzhou University, Zhengzhou, China \\ ${ }^{4}$ College of Information Engineering, China Jiliang University, Hangzhou, China \\ ${ }^{5}$ School of E-commerce \& Management Science, Zhejiang Gongshang University, Hangzhou, China \\ ${ }^{6}$ School of Economics, Fudan University, Shanghai, China \\ Correspondence should be addressed to Jingjing Chen; joyjchan@gmail.com
}

Received 20 March 2021; Accepted 29 April 2021; Published 10 May 2021

Academic Editor: Xiaokang Zhou

Copyright $\odot 2021$ Chao Li et al. This is an open access article distributed under the Creative Commons Attribution License, which permits unrestricted use, distribution, and reproduction in any medium, provided the original work is properly cited.

Defects in the textile manufacturing process lead to a great waste of resources and further affect the quality of textile products. Automated quality guarantee of textile fabric materials is one of the most important and demanding computer vision tasks in textile smart manufacturing. This survey presents a thorough overview of algorithms for fabric defect detection. First, this review briefly introduces the importance and inevitability of fabric defect detection towards the era of manufacturing of artificial intelligence. Second, defect detection methods are categorized into traditional algorithms and learning-based algorithms, and traditional algorithms are further categorized into statistical, structural, spectral, and model-based algorithms. The learning-based algorithms are further divided into conventional machine learning algorithms and deep learning algorithms which are very popular recently. A systematic literature review on these methods is present. Thirdly, the deployments of fabric defect detection algorithms are discussed in this study. This paper provides a reference for researchers and engineers on fabric defect detection in textile manufacturing.

\section{Introduction}

The influence of artificial intelligence on industrial field has far exceeded our expectations $[1,2]$. The vast number of researchers and engineers are constantly accelerating the development of industrial intelligence. In 2010, Germany put forward the Industry 4.0 framework, and the framework has been promoted and applied widely among the European Union member states. Subsequently, the United States and China put forward corresponding plans and policies for smart manufacturing of their own country. Industry 4.0 is the inevitable trend of the future development of manufacturing industry [3].

Industrial artificial intelligence is typical cross-disciplinary, which combines knowledge of mechanical, data science, network, communication, information security, and other disciplines, and it aims to use artificial intelligence algorithms to solve industrial problems and improve the efficiency and security of manufacturing [4, 5]. Towards industry 4.0, the textile manufacturing industry also needs to find its own way to adapt the manufacturing process. Textile manufacturing is a large-scale and complicated industry. The textile manufacturing process consists of a series of complex and orderly processes, mainly including spinning, weaving, dyeing, printing and finishing, and garments manufacturing. The stability and quality of the textile fabric produced by the whole production line are crucial to any enterprise [4].

There are many factors that affect the final product on the production line of textile manufacturing, such as material quality, mechanical factors, dye type, yarn size, and human factors [5]. In general, textile fabric defects refer to defects on the surface of the fabric. There are many types of 
fabric defects, most of which are caused by process problems and machine malfunctions. Defects will affect the quality of the final product, resulting in a great waste of all kinds of resources $[6,7]$. In the process of fabric manufacturing, the defects in the previous stage will affect the later stage. Therefore, early detection of fabric defects can reduce the loss of enterprises earlier and faster [8]. Therefore, effective fabric defect detection is one of the key measures for modern fabric manufacturers to control cost and enhance product value and core competence.

In modern textile manufacturing, automatic fabric defect is an important way to ensure the textile quality [9]. For long, fabric defect detection is implemented by manual visual inspection which is inadequate and expensive in the meantime. Accordingly, automatic fabric defect detection is necessary for the textile industry to reduce cost and increase productivity. The core of a complete online textile fabric defect detection system is the detection algorithms. Many researchers and engineers in this field have devoted themselves to the design of robust and efficient algorithms within the past few decades [10]. Compared to manual fabric defects detection, the automatic detection systems are more effective with higher efficiency. Fabric defect detection has been a hot research field in computer science and technology and mechanical engineering. This paper provides not only the algorithms for the researchers but also the deployment problems for the practitioners [11].

This review summarizes and classifies the methods of fabric defect detection in a broader scope. A total of more than 2,000 articles were retrieved, from which 128 articles were included in this review. The main search terms used for retrieval are "Fabric defect detection," "textile inspection," "fabric defect recognition," "automatic textile Manufacturing," etc. In addition to the traditional and classical methods mentioned in the previous reviews, this review discusses and compares the algorithms of deep learning algorithms in defect detection which are very popular in recent years. Finally, the deployment of the algorithm is also discussed; for the algorithm, the deployment is also very important to the accuracy and efficiency of the system implementation. It is hoped that this review will provide some help and suggestions for the application of AI in fabric manufacturing.

\section{Fabric Defect Detection Methods}

Fabric defect detection algorithms are roughly divided into two categories in this study, traditional algorithms and learning-based algorithms, as shown in Figure 1. Most of the traditional algorithms are based on feature engineering with prior knowledge, covering statistical, structural, spectral, and model-based methods. The learning-based algorithms can be further divided into classical machine learning algorithms and deep learning algorithms. Machine learning uses mathematical algorithms to learn and analyze data to make predictions and take decisions in the future, which has been widely employed in recent years and achieved stratifying results in various disciplines and industries.

\subsection{Traditional Algorithms}

2.1.1. Statistical Algorithms. Statistical approaches utilize the spatial distribution of gray values in images [12], such as gray-level co-occurrence matrices (GLCM), autocorrelation analysis, and fractal dimension features.

Raheja et al. present an automated fabric defect detection system utilizing GLCM. In this approach, a signal graph is constructed with GLMC statistics and interpixel distance. Then a comparison between nondefective image and test image is made. Additionally, a Gabor filter based approach is utilized to detect the defects in this study. The conclusion is made that GLCM based algorithms generate higher detection accuracies and less computational complexity [13, 14].

Anandan et al. [15] combine the GLCM and curvelet transform (CT) by extracting the eigenvector of the defect which makes the fabric defect features more evident. The experiments show the effectiveness of the proposed algorithm with comparison to GLCM and wavelet-based methods, respectively.

Kumar et al. [16] design a statistical approach for identifying defects in fabric images using eigenvalues. Using the coefficient of variation, defective portions of the fabric images are identified. This method is simple and easy to use according to the experiments in the work.

Intending to effectively detect fabric defects, Song et al. [17] calculate the membership degree of each fabric region. Utilizing the extreme point density map of the image combined with the features of the membership function region, the saliency of defect regions is obtained. The whole scheme further adopts a threshold method and morphological processing. The author states this algorithm can detect fabric defects efficiently and accurately in the presence of noise and background texture interference.

Gharsallah et al. [18] present a fabric defect detection approach utilizing an improved anisotropic diffusion filter and saliency image features. Given that the conventional anisotropic diffusion methods cannot identify the defect edge which is confused with the background texture, the improved anisotropic diffusion method combines the local gradient magnitude with a saliency map. This approach can effectively remove the texture background and retain the image defect edge.

Table 1lists several statistical algorithms used for textile fabric defect detection in brief.

2.1.2. Spectral Approach. Fourier transform, Gabor transform, wavelet transform, and discrete cosine transform [22-24] are the representative method of spectral methods. These algorithms mentioned in this survey are listed in Table 2. Fourier transform, wavelet transform, and Gabor transform-based methods have been thoroughly studied and tested on fabric defect detection applications.

Li et al. [32] propose an algorithm employing a multiscale wavelet transform and Gaussian mixture model for automated fabric defect detection. A textile fabric image was decomposed by the "Pyramid" wavelet transform and then reconstructed using thresholding method. Next, the 


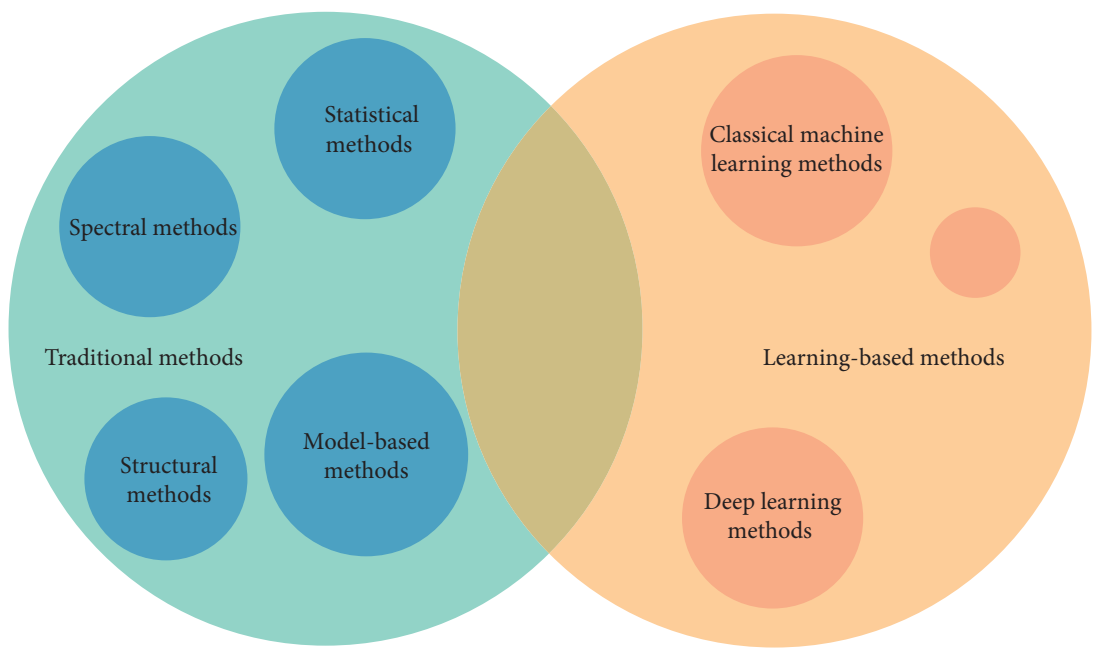

Figure 1: An overview of the defect detection methods covered in this review.

TABLE 1: Statistical algorithms for fabric defect detection.

\begin{tabular}{lccc}
\hline Author & Proposed method & Dataset & Evaluation \\
\hline Sayed [19] & Entropy filtering and minimum error thresholding & TILDA dataset & Detection success rate \\
\hline Kumari [20] & Sylvester matrix based similarity method & $\begin{array}{c}\text { KTH-TIPS-I and KTH- } \\
\text { TIPS-II }\end{array}$ & $\begin{array}{c}\text { False positives and false } \\
\text { negatives }\end{array}$ \\
\hline $\begin{array}{l}\text { Chetverikov and } \\
\text { Hanbury [21] }\end{array}$ & $\begin{array}{c}\text { Based on two fundamental structural properties, regularity } \\
\text { and local orientation (anisotropy) }\end{array}$ & $\begin{array}{c}\text { Brodatz images and } \\
\text { TILDA dataset }\end{array}$ & Detection success rate \\
\hline
\end{tabular}

Gaussian mixture model was utilized to segment the reconstructed image. The experiments demonstrate the effectiveness of the proposed algorithm for detecting and segmenting the defect images.

Rebhi et al. [33] present a fabric defect detection approach using local homogeneity information and discrete cosine transform (DCT). DCT was applied to the newly calculated homogeneity image and different energy features of all DCT blocks are then extracted. And the extracted features are fed into the feedforward neural networks classifier.

2.1.3. Structural Approach. One effective way for segmenting defective area on the patterned textile fabric image is the golden image subtraction (GIS) method. Ngan et al. proposed a method named wavelet preprocessed golden image subtraction (WGIS) [34]. Additionally, wavelet transforms, GIS, and WGIS methods are compared in this study, and the proposed WGIS achieved the best performance among them.

Jia and Liang [35] segment the fabric images into nonoverlapping regions named lattices and then the similarity of these lattices are calculated in the feature space. The proposed Isotropic Lattice Segmentation (ILS) method shows satisfying results on the box and star pattern image database. Jia et al. brought up another approach in their later study [36] on the basis of lattice segmentation and lattice templates. In this study, the lattices are segmented according to different placement rules of texture primitives that belong to different classes. The distances of undetermined lattice and lattice template are calculated, and the lattices are regarded as the defective area when the distances are larger than a certain threshold. The algorithm is further improved by adding template statistics which are learned from defectfree images in [37].

Another template-based correction approach for fabric images with periodical structure is introduced in Chang's work [38]. A fabric image is divided into lattices due to variation regularity and correction is then made to reduce the lattice misalignment. The defective lattices are first located and defect regions are segmented at a later step. Based on its assumptions, the lattice segmentation and template-based correction algorithms are more suitable for patterned fabric images.

Shi et al. [39] propose a method using low-rank decomposition of gradient information combined with structured graph. The fabric image is first divided into defect-free regions and defect regions based on the structured graph information. Adaptive thresholding is utilized during the lattice merging step. Finally, the matrix decomposition is calculated under the prior information from the segmentation results; thus the defect regions are emphasized. The presented method outperforms other methods on a standard database.

Abouelela et al. [40] design a fabric defect detection system employing simple statistical features such as median, mean, and variance. The author holds that time efficiency is crucial to any industrial procedure. Therefore, the author exchanged the complexity of simple features calculation for real-time performance. The proposed algorithm is better at detecting defects that vary drastically in the physical dimension. 
TABLe 2: Spectral algorithms for fabric defect detection.

\begin{tabular}{|c|c|c|c|}
\hline Author & Proposed method & Dataset & Evaluation \\
\hline Sulochan [25] & $\begin{array}{l}\text { Multiscale wavelet features and fuzzy C- } \\
\text { means clustering }\end{array}$ & $\begin{array}{l}\text { Real and computer-simulated } \\
\text { fabric images }\end{array}$ & Detection error rate \\
\hline $\begin{array}{l}\text { Vermaak et al. } \\
{[26]}\end{array}$ & $\begin{array}{c}\text { Dual-tree complex wavelet transform } \\
\text { (DTCWT) }\end{array}$ & TILDA dataset & Detection success rate \\
\hline $\begin{array}{l}\text { Liu and Zheng } \\
\text { [27] }\end{array}$ & $\begin{array}{l}\text { The method based on information entropy } \\
\text { and frequency domain saliency }\end{array}$ & $\begin{array}{l}\text { Database created by the } \\
\text { research associate of the } \\
\text { industrial automation research } \\
\text { laboratory }\end{array}$ & $\begin{array}{c}\text { ACC, true positive rate(TPR), false } \\
\text { positive rate(FPR), positive predictive } \\
\text { value (PPV), negative predictive value } \\
(\mathrm{NPV}) \text {, time, F-measure }\end{array}$ \\
\hline Di et al. [28] & $\begin{array}{l}\text { L0 gradient minimization method and } \\
\text { two-dimensional fractional Fourier } \\
\text { transform (2D-FRFT) for obtaining the } \\
\text { saliency map of the quaternion image }\end{array}$ & $\begin{array}{l}\text { Dataset from automation } \\
\text { laboratory fabric database of } \\
\text { Hong Kong University }\end{array}$ & $\begin{array}{l}\text { True positive (TP), false positive (FP), } \\
\text { true negative (TN), and false negative } \\
(\mathrm{FN})\end{array}$ \\
\hline Jing [29] & $\begin{array}{c}\text { Gabor preprocessed golden image } \\
\text { subtraction }\end{array}$ & $\begin{array}{c}\text { Industrial automation } \\
\text { laboratory at the University of } \\
\text { Hong Kong and the TILD } \\
\text { database }\end{array}$ & Detection success rate \\
\hline $\begin{array}{l}\text { Mohammed and } \\
\text { Alhamdani [30] }\end{array}$ & $\begin{array}{l}\text { Fuzzy back propagation neural network } \\
\text { (FBPNN) with Gabor features }\end{array}$ & Collected dataset & Detection success rate \\
\hline Yapi et al. [31] & $\begin{array}{l}\text { Using learning-based local textural } \\
\text { distributions in the contourlet domain }\end{array}$ & TILDA database & $\begin{array}{c}(\mathrm{TP}, \mathrm{FP}, \mathrm{TN} \text {, and } \mathrm{FN}) \text { local precision } \\
\left(P_{\mathrm{L}}\right) \text {, local recall }\left(R_{\mathrm{L}}\right) \text {, and local accuracy } \\
\left(\mathrm{ACC}_{\mathrm{L}}\right)\end{array}$ \\
\hline
\end{tabular}

2.1.4. Model-Based Methods. Ngan et al. propose motifbased methods for detecting defects in 2D patterned texture. This kind of methods is based on the assumption that patterned images can be divided into lattices and motifs. And further energy of moving subtraction is calculated to differentiate defective and defect-free region [41]. In order to reduce the detection rate of false positives and false negatives, the Gaussian mixture model is used to represent the energy variance value [42]. $\mathrm{K}$-means clustering is applied to the data and the convex hull of each cluster is calculated in that fitting ellipsoidal region.

Lucia et al. propose an algorithm for detecting the fabric defects in uniformly structured textile fabric images. The algorithm includes two stages: the feature extraction stage and the defect recognition stage. In the first stage, the symmetric Gabor filter bank and principal component analysis are utilized for feature extraction, and in the second stage, the Euclidean norm of features is calculated and compared for defect recognition. This algorithm is designed on a patch basis and proved to be effective on the public TILDA database [43].

For environment-friendly textiles, Shu et al. [44] adopt an algorithm based on principal component analysis and nonlocal average filtering to enhance the fabric texture and reduce the noise. A texture-based defect measurement method is used for calculating the similarity; thus defect and nondefect areas can be distinguished.

Some researchers treat the fabric defect detection problem as a one-class classification. Bu et al. $[45,46]$ propose a method based on the support vector data description (SVDD) model. In the training stage, the multiple fractal features are extracted and the optimal parameters are selected for the Gaussian kernel function. The detection results on several datasets demonstrate that this combination is effective. Bu et al. [47] also present another method using SVDD model, and the features extracted in this method are based on autoregressive (AR) spectral estimation model combined with Burg algorithm.

With analysis on statistical model-based methods, Campbell et al. [48] propose two model-based methods in their study. The first method states the maximum likelihood of image binarization. The other method is mainly for defect detection in repeated weaving patterns; thus the discrete Fourier transformation is utilized for texture analysis. In the end, a model-based clustering method is applied to delineate the defective regions.

For the detection of patterned fabrics, Tsang et al. [49] propose a method named Elo rating (ER) in line with the spirit of sports. The fabric images are divided into standardsize partitions. Matches are calculated between partitions and revised through an Elo point matrix. The defect area (partition) will win the competition as a powerful player. This presented method was tested on dot-, star-, and boxpatterned fabrics database and obtained comparable results to the most advanced method.

\subsection{Learning-Based Algorithms}

\subsubsection{Classical Machine Learning Algorithms}

(1) Dictionary Learning-Based Algorithms. Many researchers have validated the effectiveness of dictionary learning-based algorithms dealing with textile fabric defect detection problems $[50,51]$. The general steps of these algorithms: first a dictionary is learned from the training or test image, and then a fabric image without defects is reconstructed using the learned dictionary; thenceforth the detection is implemented by subtracting the reconstructed image from the test 
image. Recently, many algorithms based on low-rank representation have been brought up in the application of fabric defect inspection. In order to solve the optimization problem of the objective function, many methods reduce the lowrank decomposition problem to the nuclear minimization (NNM) problem.

$\mathrm{Li}$ et al. propose an algorithm on the basis of biological vision modeling. The biological visual saliency is modeled by low-rank representation (LRR); thus the fabric image is decomposed into salient defect regions and defect-free backgrounds [52].

Li et al. [53] model a defect-free region as a low-rank structure and the defect region as a sparse structure. Thus a fabric image can be regarded as the sum of a low-rank matrix and a sparse matrix. For dimensionality reduction, instead of singular value decomposition (SVD) on the matrix of the original image, the presented method uses eigenvalue decomposition on blocked image matrix. Therefore, this method is easy to implement and works well given that the contrast of the fabric image is sufficient.

Table 3tabulates some other dictionary learning-based algorithms using low-rank decomposition.

Shi et al. [39] point out two shortcomings of the lowrank decomposition. One is that existing low-rank decomposition models barely detect the defect regions with high gradients. And the other shortcoming is that small defect area or complex area will be incorrectly segmented given the inaccuracy of prior information. To overcome these shortcomings, Shi et al. propose a low-rank decomposition method utilizing gradient information combined with structured graph algorithm. The proposed method outperforms others on the point, box, and star databases.

Traditional machine learning algorithms, such as KNN [63] and neural network [64], are widely used in fabric defection detection problems. And feature engineering is one of the major processes in the machine learning life cycle.

Mak et al. extracted [65] four novel fractal features and employed support vector data description (SVDD), which is a support vector machine learning algorithm used for oneclass classification, for fabric defect detection in his work.

Zhang et al. propose an approach employing the radial basis function (RBF) network. Gaussian mixture model (GMM) is utilized to improve the accuracy of Gaussian RBF parameter estimation. The validity of the proposed method has been proved on multiple class datasets [66].

Tian and Li [67] propose an autoencoder-based method for fabric defect detection by exploring similarities between image patches. Utilizing the repeated texture pattern, similar nondefective patches were found for each candidate defect patch and the corresponding latent variables were weighted and combined according to which the original latent variable can be modified. Experimental results manifest the effectiveness of the proposed algorithm.

Yapi et al. [68] consider this problem as a binary problem. A compact and accurate feature set was extracted by statistical modeling of multiscale contourlet decomposition, and then a Bayesian classifier (BC) is used to classify the defect and nondefect classes. This algorithm obtained high precision detection with real-time efficiency.
Some other traditional machine learning algorithms for fabric defect detection are shown in Table 4 .

2.2.2. Deep Learning Algorithms. Recently, many researchers have applied deep learning techniques to fabric defect detection problems and have achieved satisfying results $[72,73]$ for the improvement of textile product quality and production efficiency [74]. Although deep learning methods have been proved to be powerful when dealing with segmentation and classification problems, there are still some problems in the practical application of specific industries [75]. First of all, the actual textile production line requires high real-time performance of the algorithm, which is the demand of high execution efficiency. Furthermore, compared to normal defect-free samples, the defective image data is difficult to obtain, which brings challenges to the training process of deep learning [76].

At present, the deep learning-based object detector can be classified as one-stage detectors and two-stage detectors [77]. Classical deep learning algorithms for object detection are listed in Table 5. In general, one-stage detectors have fast detection speed to meet the requirements of online detection, but the detection accuracy usually fails to meet requirements. In contrast, the two-stage algorithms have higher detection accuracy, but its detection speed is difficult to meet the real-time requirements of the algorithm in production scenes. In the field of fabric defect detection, the advantages and disadvantages of one-stage and two-stage detection algorithms are quite similar to those in other fields. The two-stage algorithm has higher accuracy but slower speed than the one-stage algorithm. In the actual application of textile industry, we hope that, under the premise of satisfying the detection accuracy, the faster the detection speed, the better. Therefore, the algorithm should be selected according to the actual application scenarios and requirements to find the balance between efficiency and accuracy.

(1) One-Stage Detection Algorithms. One-stage detection algorithm does not have a separate proposal generation phase. Typically, these algorithms treat all locations on the image as potential objects and manage to categorize each interest region into a target object or background.

The recently proposed single shot multibox detector (SSD) is a typical one-stage detector that has obtained good detection performance in object detection. This algorithm is designed based on a convolutional neural network (CNN). Some improvements have been made for the fabrics defect scenario by Liu et al. [78] and the experimental results show rationality and effectiveness.

Ouyang et al. [79] present a CNN based algorithm for on-loom fabric defect inspection. This proposed algorithm introduces a dynamic activation layer utilizing the defect probability information with a pairwise potential function to a CNN. This algorithm obtains good results dealing with the unbalanced data classification problem.

Deep convolutional neural network (DCNN) based algorithms have achieved satisfactory results on visual tasks and have been widely used in industrial scenarios. Liu et al. [80] employ DCNN to detect fabric defects with 
TABLe 3: Dictionary learning algorithms for fabric defect detection.

\begin{tabular}{|c|c|c|c|}
\hline Author & Proposed method & Dataset & Evaluation \\
\hline $\begin{array}{l}\text { Li et al. } \\
{[52]}\end{array}$ & Low-rank representation (LRR) & $\begin{array}{l}\text { (1) TILDA fabric images dataset; }(2) \\
\text { dataset from the research associate of } \\
\text { industrial automation research } \\
\text { laboratory }\end{array}$ & Precision and recall \\
\hline $\begin{array}{l}\text { Li et al. } \\
{[53]}\end{array}$ & Low-rank representation & $\begin{array}{l}500 \text { fabric images from the textile kind } \\
\text { C1 of the TILDA database }\end{array}$ & $\begin{array}{l}\text { (a) Sensitivity al } \\
\text { alarm rate (FAI }\end{array}$ \\
\hline $\begin{array}{l}\text { Gao et al. } \\
{[54]}\end{array}$ & Gabor filter and ten & $\begin{array}{c}\text { Dataset from the research associate of } \\
\text { industrial automation research } \\
\text { laboratory }\end{array}$ & $\begin{array}{c}\text { Receiver operating characteristic } \\
\text { curve (ROC) }\end{array}$ \\
\hline $\begin{array}{l}\text { Shi et al. } \\
{[39,47]}\end{array}$ & $\begin{array}{l}\text { Low-rank decomposition with gradient } \\
\text { information }\end{array}$ & $\begin{array}{c}\text { Dataset from the research asso } \\
\text { industrial automation resea } \\
\text { laboratory }\end{array}$ & TPR, FPR, PPV, NPV \\
\hline $\begin{array}{l}\text { Liu et al. } \\
{[55-57]}\end{array}$ & $\begin{array}{l}\text { Multi-scale convolutional neural network and } \\
\text { low-rank decomposition model }\end{array}$ & $\begin{array}{l}\text { (1) TILDA fabric images dataset; }(2) \\
\text { dataset from the research associate of } \\
\text { industrial automation research } \\
\text { laboratory }\end{array}$ & $\begin{array}{l}\text { Means and standard deviations of } \\
\text { average precisions, recalls, F- } \\
\text { measure, and mean absolute error } \\
\text { (MAE) }\end{array}$ \\
\hline $\begin{array}{l}\text { Mo et al. } \\
{[58]}\end{array}$ & $\begin{array}{c}\text { Weighted double-low-rank decomposition } \\
\text { method (WDLRD) to treat the matrix singular } \\
\text { values differently by assigning different } \\
\text { weights }\end{array}$ & $\begin{array}{c}\text { Database is from the research associate } \\
\text { of industrial automation research } \\
\text { laboratory, HKBU }\end{array}$ & $\begin{array}{l}\text { Visual defect locating results, the } \\
\text { metrics of false alarm, recall, } \\
\text { precision, accuracy, and F-measure }\end{array}$ \\
\hline $\begin{array}{l}\text { Li et al. } \\
\text { [59] }\end{array}$ & $\begin{array}{l}\text { Low-rank decomposition of multichannel } \\
\text { feature matrices }\end{array}$ & $\begin{array}{l}\text { (1) TILDA fabric images dataset; }(2) \\
\text { dataset from the research associate of } \\
\text { industrial automation research } \\
\text { laboratory }\end{array}$ & $\begin{array}{c}\text { ROC curves and precision-recall (PR) } \\
\text { curves }\end{array}$ \\
\hline $\begin{array}{l}\text { Yang et al. } \\
{[60]}\end{array}$ & $\begin{array}{l}\text { Sparse and dense mixed low-rank } \\
\text { decomposition }\end{array}$ & $\begin{array}{l}\text { Real-world samples of } 512 * 512 \text { with } \\
\text { 256-gray levels }\end{array}$ & Saliency map (qualitative) \\
\hline $\begin{array}{l}\text { Wang } \\
\text { et al. [61] }\end{array}$ & $\begin{array}{l}\text { A randomized low-rank and sparse matrix } \\
\text { decomposition model named GoDec }\end{array}$ & $\begin{array}{l}\text { Fabric image dataset collected by Dr. } \\
\text { Henry Y. T. Ngan [62] }\end{array}$ & Precision, recall, and F-measure \\
\hline
\end{tabular}

TABLE 4: Traditional machine learning algorithms for fabric defect detection.

\begin{tabular}{lccc}
\hline Author & Proposed method & Dataset & Evaluation \\
\hline Wang et al. [63] & $\begin{array}{c}\text { Multiview stereo vision (MVS) and bag-of- } \\
\text { features (BOF), K-nearest neighbor (KNN) } \\
\text { algorithm }\end{array}$ & Collected dataset & Detection success rate \\
\hline $\begin{array}{l}\text { Priyanka and } \\
\text { Manish [69] }\end{array}$ & Artificial neural networks (ANN) & Collected dataset & Detection success rate \\
\hline $\begin{array}{l}\text { Bumrungkun } \\
\text { [70] }\end{array}$ & $\begin{array}{c}\text { Snake active contour and support vector } \\
\text { machines }\end{array}$ & Collected dataset & $\begin{array}{c}\text { Recognition accuracy } \\
\text { detection success rate }\end{array}$ \\
\hline Zhang et al. [71] & $\begin{array}{c}\text { L0 gradient minimization (LGM) and the } \\
\text { fuzzy c-means (FCM) method to detect } \\
\text { various fabric defects with diverse textures }\end{array}$ & $\begin{array}{c}\text { Images from the automation laboratory } \\
\text { sample database of Hong Kong University, } \\
\text { TILDA textile texture database, and Guang } \\
\text { Dong Esquel Textiles }\end{array}$ & $\begin{array}{c}\text { ACC, TPR, FPR, PPV, } \\
\text { and IOU (intersection } \\
\text { over union) }\end{array}$ \\
\hline
\end{tabular}

complicated textures. This proposed approach is particularly designed for real textile production environment with limited resources. A series of improvements have been done to make the detection more effective. Zhou et al. propose an efficient DCNN architecture focusing on the problem of fabric defect detection, called Efficient Defect Detectors (EDDs) [81]. To extract more low-level features, EDDs adjust the input resolution, depth, and width using a scaling strategy. The improvement proved to be effective when compared with existing fabric defect detection algorithms.

$\mathrm{Xu}$ et al. [82] propose a novel detection network named de-deformation defect detection network (D4Net). This
TABLE 5: Deep learning algorithms for detecting object.

\begin{tabular}{lc}
\hline One-stage detectors & Two-stage detectors \\
\hline YOLO & Faster RCNN \\
SSD & Mask RCNN \\
YOLOv2/v3/v4 & Cascade RCNN \\
RefineDet & FPN \\
RetinaNet & R-FCN \\
\hline
\end{tabular}

model is composed of reference generation, de-deformation network, and marginal loss. The most suitable reference is selected and paired with the input image and then is sent into the de-deformation network. The dissimilarities are 
calculated and enhanced by the marginal loss. Experiments on a large industrial database containing $67 \mathrm{~K}$ images have been done and the results show that the algorithm outperforms other algorithms especially for large pattern fabric images.

Peng et al. put forward a detection algorithm called Priori Anchor Convolutional Neural Network (PRAN-Net) to fix this problem. Feature Pyramid Network (FPN) is utilized to selected multiscale feature maps and then sparse priori anchors are generated based on ground truth boxes [83].

Li et al. [84] propose an architecture using several microarchitectures. The microarchitecture is constructed of multiscale analysis, filter factorization, multilocation pooling, and parameters reduction, thus making the network a compact one. With the small model size, the proposed net worked well on fabric defect detection.

(2) Two-Stage Detection Algorithms. With regard to twostage detectors, a sparse set of proposals is generated in the first stage and in the second stage, and the features of generated proposals are sent into DCNN for prediction results. As a remarkable two-stage detector, Faster R-CNN is an object detection model that improves on Fast R-CNN by utilizing a region proposal network (RPN) with the CNN model. Some researchers utilize the modified Faster R-CNN model for fabric defect detection [85-87]. Jun et al. [88] propose a framework that utilized the Inception-V1 model and LeNet-5 model. This approach includes local defect prediction in the first stage and global defect recognition in the second stage.

Table 6 lists some other deep learning algorithms utilized in textile fabric defect applications.

In recent years, Generative Adversarial Networks (GANs) have attracted a lot of attention [97, 98]. GANs and related algorithms have been widely used in a range of computer vision and computer graphics applications, such as image synthesis and video generation. GAN based fabric defect detection algorithms can automatically adapt to different fabric textures by learning existing fabric defect samples [99]. Liu et al. design a deep semantic segmentation network to detect fabric defects. They train a multistage GAN model to synthesize reasonable defect samples from nondefect samples. The performance of the method was verified by comprehensive experiments on all sorts of typical fabric image samples.

Le et al. [100] utilize Wasserstein generative adversarial nets (WGANs) combined with transfer learning techniques and multimodel ensembling framework. The effectiveness of the proposed scheme is demonstrated on unbalanced and rare datasets of images with defects.

Inspired by biological visual perception mechanism, Zhao et al. [101] describe a CNN model based on visual longshort-term memory (VLSTM). Three types of features, visual perception features, visual short-term memory (VSTM) features, and visual long-term memory (VLTM) features, are extracted by stacked convolutional autoencoders, a shallow $\mathrm{CNN}$, and nonlocal neural networks, respectively. Experiments have been done on three public datasets and results show that the proposed algorithm is comparable to state-ofthe-art algorithms.

Traditional saliency detection models usually rely on hand-crafted features to capture the information of global context and local details. Researchers add the attention mechanism to deal with fabric defect detection problems. Wang et al. [102] propose a deep saliency detection model that incorporated self-attention mechanism into a $\mathrm{CNN}$ for fabric defect detection. Multiscale feature maps are generated from a fully convolutional network, and a self-attention module is used to coordinate the dependence between the features of different layers. The self-attention mechanism in this algorithm proved to be very effective with complex or blurred defects.

Some studies combine the traditional and deep learning methods. Wang et al. [103] extracted global deep features using $\mathrm{CNN}$ in combination with handcrafted low-level features, and nonconvex robust PCA regularized by nonconvex total variation are employed to data processing and noise reduction. Then a segmentation algorithm is used to segment the saliency map to obtain the defect area.

\section{Application and Deployment}

When it comes to the deployment phase, there will be a lot of engineering implementation problems [104, 105]. Realizing an intelligent textile system in the real textile manufacturing process covers many aspects, involving the Internet of Things (IoT) [106], cyber-physical systems (CPS) [107], and more $[108,109]$.

3.1. Hardware Selection of Detection System. The basic components of image acquisition system are very important for the deployment work and therefore the hardware selection is critical for subsequent detection work [110]. Hardware such as cameras, lens, lights, and frame grabber is an important factor. Different hardware corresponds to different subsequent algorithms. For instance, Yildiz et al. [111] present a new fabric defect detection method for using a thermal camera. Fabric images obtained by the thermal imaging camera have their own image characteristics. The algorithm can be designed utilizing thermal differences between defect and defect-free areas and to improve the detection accuracy and efficiency while reducing the cost.

Different from visual inspection systems, Fang et al. [112] introduce a tactile inspection system for fabric defect detection. The system design is mainly based on a visual tactile sensor, which consists of several LEDs, a camera, and an elastic sensing layer. This system captures detailed information of surface structure neglecting of color and pattern; thus the algorithm designed in this study is mainly based on the structural information obtained from the tactile sensor.

In addition, the conveyor belt used for conveying cloth on the production line will also affect the image taking speed [113]. Therefore, the hardware selection of the entire system needs to be considered as a whole. 
TABLE 6: Deep learning-based algorithms for fabric defect detection.

\begin{tabular}{|c|c|c|c|c|}
\hline Author & Proposed or tested model & Categorize & Dataset & Evaluation \\
\hline Jing et al. [89] & Mobile-Unet & One-stage & $\begin{array}{l}\text { Benchmark databases, the fabric images } \\
\text { database (FID), and yarn dyed fabric } \\
\text { images (YFI), in which all images are } \\
\text { manually annotated segmentation }\end{array}$ & Pixel accuracy (PA) and IoU \\
\hline $\begin{array}{l}\text { Hong-wei } \\
\text { hang [90] }\end{array}$ & YOLOV2 & One-stage & Collected dataset (276) & IOU, recall, and precision \\
\hline $\begin{array}{l}\text { Young-Joo } \\
\text { Han [91] }\end{array}$ & $\begin{array}{c}\text { Stacked convolutional } \\
\text { autoencoders }\end{array}$ & One-stage & Synthetic and collected dataset & Recall, precision, and F-score \\
\hline $\begin{array}{l}\text { Xinying } \mathrm{He} \\
{[92]}\end{array}$ & $\begin{array}{l}\text { Adaptive method based on } \\
\text { DenseNet-SSD }\end{array}$ & One-stage & Collected dataset (2072) & $\begin{array}{l}\text { Calculate localization loss } \\
\text { (loc) and confidence loss } \\
\text { (conf) }\end{array}$ \\
\hline $\begin{array}{l}\text { Mohammed } \\
\text { et al. [93] }\end{array}$ & $\begin{array}{c}\text { A multilayer perceptron with a } \\
\text { Levenberg-Marquardt (LM) } \\
\text { algorithm }\end{array}$ & One-stage & Collected dataset (217) & $\begin{array}{l}\text { Specificity, accuracy, and } \\
\text { sensitivity }\end{array}$ \\
\hline $\begin{array}{l}\text { Shuang mei } \\
{[94]}\end{array}$ & $\begin{array}{c}\text { Multiscale convolutional } \\
\text { denoising autoencoder network } \\
\text { model }\end{array}$ & One-stage & $\begin{array}{l}\text { Four datasets: fabrics, KTH-TIPS, } \\
\text { Kylberg texture, and ms-texture }\end{array}$ & $\begin{array}{l}\text { Recall, precision, and F1- } \\
\text { measure }\end{array}$ \\
\hline $\begin{array}{l}\text { Huosheng Xie } \\
\text { [95] }\end{array}$ & Improved RefineDet & One-stage & $\begin{array}{c}\text { TILDA dataset, Hong Kong patterned } \\
\text { textures database, and DAGM2007 } \\
\text { dataset }\end{array}$ & $\begin{array}{l}\text { Precision }(\mathrm{P}) \text {, recall } \mathrm{I}, \mathrm{F} 1- \\
\text { score, mean average precision } \\
(\mathrm{mAP}), \text { model parameter } \\
\text { (param.) }\end{array}$ \\
\hline $\begin{array}{l}\text { Yanqing } \\
\text { Huang [96] }\end{array}$ & $\begin{array}{l}\text { Segmentation network and } \\
\text { decision network }\end{array}$ & Two-stage & $\begin{array}{l}\text { Dark redfFabric (DRF), light blue fabric } \\
\text { (LBF) and patterned texture fabric (PTF) }\end{array}$ & $\begin{array}{c}\text { Frames per second (FPS) } \\
\text { Avg-IoU and Avg-P }\end{array}$ \\
\hline
\end{tabular}

3.2. Dataset. This review lists a lot of learning-based algorithms. Although this type of method is very effective, it requires a large number of labeled fabric images with defects. However, it is very difficult to collect a fair amount of fabric defect image data in industrial scenes [114]. Therefore, many researchers employ semisupervised and unsupervised learning algorithms for the detection [115]. In addition, some studies utilize nondefect image data and synthetic defective image data generated by using defect characteristics based on expert knowledge [91]. Chen et al. [116] propose a data augmentation method based on automatic image acquisition. Different image acquisition angles, various acquisition scenes, and random illumination conditions are designed for image collection as a simulation under the actual textile production scene.

In addition, the diversity of fabric defects and unbalanced categories bring actual challenges to fabric defect detection [86]. For instance, the multiscale defects in the fabric image will greatly increase the complexity and computation of the model; thus the designed fabric defect detection model of appropriate size must be able to meet the multiscale target detection.

3.3. Real Time of the Algorithm. Generally, the actual fabric defect detection task is implemented online on a platform with limited computing power. Thus the algorithms of online defect detection systems need to be accurate, efficient, and robust [117]. Therefore, the robustness and efficiency of the algorithm are critical to the actual production line. The computational cost of different algorithms is a critical consideration.
However, there are many other problems that exist in the textile production scenario. In order to obtain better image data and detection results, most existing algorithms require textile to be flattened. Therefore, some scholars design defect detection algorithms specifically for textiles with uneven and diverse shapes [118]. This consideration is closer to real-world settings.

Shunji et al. [119] design a detection method for tubular knitted fabric which is produced by a circular knitting machine. Vertical defects in circular knitted fabrics are caused by damaged needles. Once a vertical defect occurs during knitting, it will continue to exist unless the damaged needle is replaced with a new one.

In general, there is no real-time quality control system that can guarantee the quality in the production of noncrimp fabrics. The embedded system proposed by Schmitt et al. [120] ensures that all steps of image acquisition, processing, and evaluation can be executed in real time. The advantage of this proposed system is that real-time, accurate, and robust performance of the algorithm is ensured by detecting fiber orientation under industrial conditions.

In practical applications, fabric defect detection algorithms must not only ensure detection accuracy but also guarantee its applicability to hardware platforms with limited resources. Currently, the accuracy of existing detection models is low. This is due in large part to multiscale defects in the fabric image; so, the fabric defect detection model must be able to meet multiscale object detection. However, even the best model is still troubled by the large size of the problem. Therefore, we must consider ways to reduce the size of the model. Inspired by the successful use of deep convolutional neural networks (DCNN) for target detection, we propose a wide-and-light network structure called WALNet. 


\section{Discussion}

Fabric defects correspond to defects on the surface of the textile fabric. Most fabric defects are caused by machine or process faults and malfunctions. The existence of fabric defects greatly reduces the sale and use of textiles. Textile manufacturing companies need to upgrade equipment and technology to maintain growth and competitiveness.

The sensing, storage, and computing capabilities of automated fabric detection systems based on computer vision will continue to improve. The development of hardware and algorithms will greatly affect the accuracy of detection and the ease of deployment.

Besides the fabric defect detection phase discussed in this survey, there is a lot of work that needs to be done during the whole textile manufacturing process. A lot of research works have been proposed for yarn production, fabric manufacturing, and finishing process utilizing learningbased methods. Huynh [121] proposes an online fabric defect prediction method based on the back propagation neural network models. The acquired data is collected in the form of time series and then converted into regional data based on the control chart. The proposed model can predict the defect types in advance and thus can reduce the workload of quality control in the production process.

In the future, more work needs to be done in the process of moving towards Industry $4.0[122,123]$. Smart manufacturing integrates various technologies, covering robotics, CPS, IoT, big data analytics [124], and cloud computing. CPS is an engineering system that seamlessly integrates physical and computational components [125]. Adding artificial intelligence, big data analysis, and cloud services to the IoT ecosystem is the key development direction of CPS in the future.

\section{Conclusions}

This paper presents a systematic literature review on automatic fabric defect detection methods of the textile industry smart manufacturing. All the methods covered in this work are roughly classified into two main categories, namely, traditional algorithms and learning-based algorithms. There are no clear boundaries between the different categories. To realize a better detection result, the researchers often combine different algorithms. The research results of this survey also confirmed that better results can be obtained by combining different methods and thus provide suggestions and ideas for further research. Accurate, efficient, and robust fabric defect detection algorithms are necessary to develop fully automated web detection systems.

The automatic textile fabric defect detection technology based on computer vision has attracted great attention of researchers. With the development of new object detection algorithms, computational capabilities, and sensor technology and industry, computer-vision based textile defect detection techniques will continue to evolve at a high speed.

\section{Conflicts of Interest}

The authors declare no conflicts of interest.

\section{Authors' Contributions}

Research plan was carried out by Jingjing Chen and Chao Li; original draft preparation was performed by Chao $\mathrm{Li}$; reviewing and editing were performed by Jingjing Chen, Jun $\mathrm{Li}$, Yafei Li, Lingmin He, and Xiaokang Fu.

\section{Acknowledgments}

This research was funded by Zhejiang Provincial Basic Public Welfare Research Program, grant number LQ20F020024.

\section{References}

[1] J. Lee, Industrial AI, Springer Singapore, Singapore, 2020.

[2] K. Yan, L. Liu, Y. Xiang, and Q. Jin, "Guest editorial: AI and machine learning solution cyber intelligence technologies: new methodologies and applications," IEEE Transactions on Industrial Informatics, vol. 16, no. 10, pp. 6626-6631, 2020.

[3] K.-D. Thoben, S. Wiesner, S. Wiesner, and T. Wuest, "“Industrie 4.0" and smart manufacturing-a review of research issues and application examples," International Journal of Automation Technology, vol. 11, no. 1, pp. 4-16, 2017.

[4] A. Kumar, "Computer vision-based fabric defect analysis and measurement," in Computer Technology for Textiles and Apparel: Woodhead Publishing Series in Textiles, J. Hu, Ed., pp. 45-65, Woodhead Publishing, Cambridge, UK, 2011.

[5] X. Xu, D. Cao, Y. Zhou, and J. Gao, "Application of neural network algorithm in fault diagnosis of mechanical intelligence," Mechanical Systems and Signal Processing, vol. 141, p. 106625, 2020.

[6] J. Bullon, A. González Arrieta, A. Hernández Encinas et al., "Manufacturing processes in the textile industry. Expert Systems for fabrics production," ADCAIJ: Advances in Distributed Computing and Artificial Intelligence Journal, vol. 6, no. 4, pp. 15-23, 2017.

[7] M. Sibly Sadik, Defects of Woven Fabrics and Their Remedies, Bachelor of Science, Department of Textile Engineering, Daffodil International University, Dhaka, Bangladesh, 2014.

[8] K. Singh, J. Kaleka, and J. Kaleka, "Identification and classification of fabric defects," International Journal of Advanced Research, vol. 4, no. 8, pp. 1137-1141, 2016.

[9] L. Song, R. Li, and S. Chen, "Fabric defect detection based on membership degree of regions," IEEE Access, vol. 99, p. 1, 2020.

[10] P. M. Mahajan, S. R. Kolhe, and P. M. Patil, "A review of automatic fabric defect detection techniques," Advances in Computational Research, vol. 1, no. 2, pp. 18-29, 2009.

[11] H. Y. T. Ngan, G. K. H. Pang, and N. H. C. Yung, "Automated fabric defect detection-A review," Image and Vision Computing, vol. 29, no. 7, pp. 442-458, 2011.

[12] M. T. Habib, S. B. Shuvo, M. S. Uddin et al., "Automated textile defect classification by bayesian classifier based on statistical features," in Proceedings of the 2016 International Workshop on Computational Intelligence (IWCI), Dhaka, Bangladesh, December 2016.

[13] J. L. Raheja, S. Kumar, and A. Chaudhary, "Fabric defect detection based on GLCM and Gabor filter: a comparison," Optik, vol. 124, no. 23, pp. 6469-6474, 2013. 
[14] J. L. Raheja, B. Ajay, and A. Chaudhary, "Real time fabric defect detection system on an embedded DSP platform," Optik, vol. 124, no. 21, pp. 5280-5284, 2013.

[15] R. S. Sabeenian, "Fabric defect detection using discrete curvelet transform," Procedia Computer Science, vol. 133, pp. 1056-1065, 2018.

[16] P. Senthil Kumar and H. Hafedh, "Detection of defects in knitted fabric images using Eigen values," International Journal Computer Science Engineering-IJASCSE, vol. 2, no. 3, pp. 7-10, 2013.

[17] L. Song, R. Li, and S. Chen, "Fabric defect detection based on membership degree of regions," IEEE Access, vol. 99, p. 1, 2020.

[18] M. B. Gharsallah and E. B. Braiek, "A visual attention system based anisotropic diffusion method for an effective textile defect detection," Journal of the Textile Institute, vol. 8, pp. 1-15, 2020.

[19] M. S. Sayed, "Robust fabric defect detection algorithm using entropy filtering and minimum error thresholding," in Proceedings of the 2016 IEEE 59th International Midwest Symposium on Circuits and Systems (MWSCAS), Abu Dhabi, UAE, October 2016.

[20] R. M. L. N. Kumari, "Sylvester matrix based similarity estimation method for automation of defect detection in textile fabrics," Journal of Sensors, vol. 2021, Article ID 6625421, 11 pages, 2021.

[21] D. Chetverikov and A. Hanbury, "Finding defects in texture using regularity and local orientation," Pattern Recognition, vol. 35 , no. 10, pp. 2165-2180, 2002.

[22] L. Chen, S. Zeng, Q. Gao et al., "Adaptive gabor filtering for fabric defect inspection," Journal of Compurters, vol. 31, no. 2, pp. 45-55, 2020.

[23] J. Zhang, Y. Li, and H. Luo, "Defect detection in textile fabrics with optimal Gabor filter and BRDPSO algorithm," Journal of Physics: Conference Series, vol. 1651, p. 012073, 2020.

[24] B. A. TanveerSajid, "Fabric defect detection in textile images using gabor filter," IOSR Journal of Electrical and Electronics Engineering, vol. 3, no. 2, 2012.

[25] H. C. Sulochan, "Fabric weave pattern detection based on fuzzy clustering and texture orientation features in wavelet domain," Journal of Textile Science \& Engineering, vol. 8, no. 6, 2018.

[26] N. Vermaak, P. Nsengiyumva, and N. Luwes, "Using the dual-tree complex wavelet transform for improved fabric defect detection," Journal of Sensors, vol. 2016, Article ID 9794723, 8 pages, 2016.

[27] G. Liu and X. Zheng, "Fabric defect detection based on information entropy and frequency domain saliency," Visual Computer, vol. 24, 2020.

[28] L. Di, H. Long, and J. Liang, "Fabric defect detection based on illumination correction and visual salient features," Sensors, vol. 20, no. 18, 2020.

[29] J.-F. Jing, S. Chen, and P.-F. Li, "Fabric defect detection based on golden image subtraction," Coloration Technology, vol. 133, 2017.

[30] I. S. Mohammed and I. M. Alhamdani, "A fuzzy system for detection and classification of textile defects to ensure the quality of fabric production," International Journal of Electrical and Computer Engineering, vol. 9, no. 5, 2019.

[31] D. Yapi, M. S. Allili, and N. Baaziz, "Automatic fabric defect detection using learning-based local textural distributions in the contourlet domain," IEEE Transactions on Automation Science and Engineering, vol. 15, no. 3, pp. 1014-1026, 2018.
[32] P. Li, H. Zhang, J. Jing, R. Li, and J. Zhao, "Fabric defect detection based on multi-scale wavelet transform and Gaussian mixture model method," The Journal of The Textile Institute, vol. 106, no. 6, pp. 587-592, 2015.

[33] A. Rebhi, I. Benmhammed, S. Abid, and F. Fnaiech, "Fabric defect detection using local homogeneity analysis and neural network," Journal of Photonics, vol. 2015, Article ID 376163, 9 pages, 2015.

[34] H. Y. T. Ngan, G. K. H. Pang, S. P. Yung, and M. K. Ng, "Wavelet based methods on patterned fabric defect detection," Pattern Recognition, vol. 38, no. 4, pp. 559-576, 2005.

[35] L. Jia and J. Liang, "Fabric defect inspection based on isotropic lattice segmentation," Journal of the Franklin Institute, vol. 354, no. 13, pp. 5694-5738, 2017.

[36] L. Jia, J. Zhang, S. Chen, and Z. Hou, "Fabric defect inspection based on lattice segmentation and lattice templates," Journal of the Franklin Institute, vol. 355, no. 15, pp. 77647798, 2018.

[37] L. Jia, C. Chen, S. Xu, and J. Shen, "Fabric defect inspection based on lattice segmentation and template statistics," Information Sciences, vol. 512, pp. 964-984, 2020.

[38] X. Chang, C. Gu, J. Liang et al., "Fabric defect detection based on pattern template correction," Mathematical Problems in Engineering, vol. 2018, Article ID 3709821, 17 pages, 2018.

[39] B. Shi, J. Liang, L. Di, C. Chen, and Z. Hou, "Fabric defect detection via low-rank decomposition with gradient information and structured graph algorithm," Information Sciences, vol. 546, pp. 608-626, 2021.

[40] A. Abouelela, H. M. Abbas, H. Eldeeb, A. A. Wahdan, and S. M. Nassar, "Automated vision system for localizing structural defects in textile fabrics," Pattern Recognition Letters, vol. 26, no. 10, pp. 1435-1443, 2005.

[41] H. Y. T. Ngan, G. K. H. Pang, and N. H. C. Yung, "Motifbased defect detection for patterned fabric," Pattern Recognition, vol. 41, no. 6, pp. 1878-1894, 2008.

[42] H. Y. T. Ngan, G. K. H. Pang, and N. H. C. Yung, "Ellipsoidal decision regions for motif-based patterned fabric defect detection," Pattern Recognition, vol. 43, no. 6, pp. 2132-2144, 2010.

[43] L. Bissi, G. Baruffa, P. Placidi, E. Ricci, A. Scorzoni, and P. Valigi, "Automated defect detection in uniform and structured fabrics using Gabor filters and PCA," Journal of Visual Communication and Image Representation, vol. 24, no. 7, pp. 838-845, 2013.

[44] Y. Shu, L. Zhang, D. Zuo et al., "Analysis of texture enhancement methods for the detection of eco-friendly textile fabric defects," Journal of Intelligent \& Fuzzy Systems, pp. 1-11, 2021.

[45] H.-G. Bu, J. Wang, and X.-B. Huang, "Fabric defect detection based on multiple fractal features and support vector data description," Engineering Applications of Artificial Intelligence, vol. 22, no. 2, pp. 224-235, 2009.

[46] H.-G. Bu, X.-B. Huang, J. Wang, and X. Chen, "Detection of fabric defects by auto-regressive spectral analysis and support vector data description," Textile Research Journal, vol. 80, no. 7, pp. 579-589, 2010.

[47] B. Shi, J. Liang, L. Di et al., "Fabric Defect Detection via LowRank Decomposition With Gradient Information," IEEE Access, vol. 99, p. 1, 2019.

[48] J. G. Campbell, C. Fraley, D. Stanford, F. Murtagh, and A. E. Raftery, "Model-based methods for textile fault detection," International Journal of Imaging Systems and Technology, vol. 10, no. 4, pp. 339-346, 1999. 
[49] C. S. C. Tsang, H. Y. T. Ngan, and G. K. H. Pang, "Fabric inspection based on the Elo rating method," Pattern Recognition, vol. 51, pp. 378-394, 2016.

[50] L. Tong, W. K. Wong, and C. K. Kwong, "Fabric defect detection for apparel industry: a nonlocal sparse representation approach," IEEE Access, vol. 5, pp. 5947-5964, 2017.

[51] X. Kang and E. Zhang, "A universal and adaptive fabric defect detection algorithm based on sparse dictionary learning," IEEE Access, vol. 99, p. 1, 2020.

[52] C. Li, G. Gao, Z. Liu, M. Yu, and D. Huang, "Fabric defect detection based on biological vision modeling," IEEE Access, vol. 6, pp. 27659-27670, 2018.

[53] P. Li, J. Liang, X. Shen, M. Zhao, and L. Sui, "Textile fabric defect detection based on low-rank representation," Multimedia Tools and Applications, vol. 78, no. 1, pp. 99-124, 2019.

[54] G. Gao, C. Liu, Z. Liu et al., Eds., in Proceedings of the 2017 4th IAPR Asian Conference on Pattern Recognition (ACPR), Nanjing, China, November 2017.

[55] Z. Liu, B. Wang, C. Li et al., "Fabric defect detection based on deep-feature and low-rank decomposition," Journal of Engineered Fibers \& Fabrics, vol. 15, 2020.

[56] Z. Liu, B. Wang, C. Li et al., "Fabric defect detection algorithm based on convolution neural network and low-rank representation," in Proceedings of the 2017 4th IAPR Asian Conference on Pattern Recognition (ACPR), Nanjing, China, November 2018.

[57] Z. Liu, L. Yan, C. Li, Y. Dong, and G. Gao, "Fabric defect detection based on sparse representation of main local binary pattern," International Journal of Clothing Science and Technology, vol. 29, no. 3, pp. 282-293, 2017.

[58] D. Mo, W. K. Wong, Z. Lai, and J. Zhou, "Weighted doublelow-rank decomposition with application to fabric defect detection," IEEE Transactions on Automation Science and Engineering, vol. 99, pp. 1-21, 2020.

[59] C. Li, C. Liu, G. Gao, Z. Liu, and Y. Wang, "Robust low-rank decomposition of multi-channel feature matrices for fabric defect detection," Multimedia Tools and Applications, vol. 78, no. 6, pp. 7321-7339, 2019.

[60] Y. Yang, J. Wang, Z. Liu et al., "Fabric defect detection method based on sparse and dense mixed low-rank decomposition," in Proceedings of the 2018 International Conference on Security, Pattern Analysis, and Cybernetics (SPAC), Shenzhen, China, December 2018.

[61] J. Wang, Q. Li, J. Gan et al., "Fabric defect detection based on improved low-rank and sparse matrix decomposition," in Proceedings of the 2017 IEEE International Conference on Image Processing (ICIP), Beijing, China, September 2017.

[62] Y. Ngan, G. Pang, S. Yung et al., "Defect detection on patterned jacquard fabric," in Proceedings of the 32nd Applied Image Pattern Recognition Workshop (AIPR 2003), Image Data Fusion, Washington, DC, USA, October 2003.

[63] Y. Wang, N. Deng, and B. Xin, "Investigation of 3D surface profile reconstruction technology for automatic evaluation of fabric smoothness appearance," Measurement, vol. 166, p. $108264,2020$.

[64] S. Kulkarni, K. Jojare, V. Bhosale, and P. Arude, "Textile fabric defect detection," IJARCCE, vol. 5, no. 12, pp. 476-478, 2016.

[65] K. L. Mak, P. Peng, and K. F. C. Yiu, "Fabric defect detection using morphological filters," Image and Vision Computing, vol. 27 , no. 10, pp. 1585-1592, 2009.

[66] Y. Zhang, Z. Lu, and J. Li, "Fabric defect classification using radial basis function network," Pattern Recognition Letters, vol. 31, no. 13, pp. 2033-2042, 2010.
[67] H. Tian and F. Li, "Autoencoder-based fabric defect detection with cross- patch similarity," in Proceedings of the 2019 16th International Conference on Machine Vision Applications (MVA), Tokyo, Japan, May 2019.

[68] D. Yapi, M. Mejri, M. S. Allili, and N. Baaziz, "A learningbased approach for automatic defect detection in textile images," IFAC-PapersOnLine, vol. 48, no. 3, pp. 2423-2428, 2015.

[69] V. Priyanka and K. Manish, "Fabric defect inspection system using neural network," International Journal of Multidisciplinary Research and Development, vol. 2, no. 4, pp. 569-573, 2015.

[70] P. Bumrungkun, "Defect detection in textile fabrics with snake active contour and support vector machines," Journal of Physics: Conference Series, vol. 1195, Article ID 012006, 2019.

[71] H. Zhang, J. Ma, J. Jing, and P. Li, "Fabric defect detection using L0 gradient minimization and fuzzy C-means," $A p$ plied Sciences, vol. 9, no. 17, p. 3506, 2019.

[72] D. Siegmund, B. Fu, A. Jose-Garcia et al., "Study and research on detection of fiber defects using keypoints and deep learning," International Journal of Pattern Recognition \& Artificial Intelligence, 2020.

[73] S. Das, A. Wahi, S. Keerthika, and N. Thulasiram, "Defect analysis of textiles using artificial neural network," Current Trends in Fashion Technology \& Textile Engineering, vol. 6, no. $1,2020$.

[74] S. Barua, H. Patil, P. D. Desai et al., "Deep learning-based smart colored fabric defect detection system," Applied Computer Vision and Image Processing, pp. 212-219, 2020.

[75] X. Zhou, Y. Li, and W. Liang, "CNN-RNN based intelligent recommendation for online medical pre-diagnosis support," IEEE/ACM Transactions on Computational Biology and Bioinformatics, p. 1, 2020.

[76] Y. Li, W. Zhao, and J. Pan, "Deformable patterned fabric defect detection with Fisher criterion-based deep learning," IEEE Transactions on Automation Science and Engineering, vol. 14, no. 2, pp. 1256-1264, 2017.

[77] X. Wu, D. Sahoo, and S. C. H. Hoi, "Recent advances in deep learning for object detection," Neurocomputing, vol. 396, pp. 39-64, 2020.

[78] Z. Liu, S. Liu, and C. Li, "Fabric defects detection based on SSD," in Proceedings of the 2nd International Conference on Graphics and Signal Processing: ICGSP'18, et al. , Sydney, Australia, October 2018.

[79] W. Ouyang, B. Xu, J. Hou, and X. Yuan, "Fabric defect detection using activation layer embedded convolutional neural network," IEEE Access, vol. 7, pp. 70130-70140, 2019.

[80] Z. Liu, C. Zhang, C. Li et al., "Fabric defect recognition using optimized neural networks," Journal of Engineered Fibers and Fabrics, vol. 14, 2019.

[81] T. Zhou, J. Zhang, H. Su, W. Zou, and B. Zhang, "EDDs: a series of Efficient Defect Detectors for fabric quality inspection," Measurement, vol. 172, p. 108885, 2021.

[82] X. Xu, J. Chen, H. Zhang, and W. W. Y. Ng, "D4Net: dedeformation defect detection network for non-rigid products with large patterns," Information Sciences, vol. 547, pp. 763-776, 2021.

[83] P. Peng, Y. Wang, C. Hao, Z. Zhu, T. Liu, and W. Zhou, "Automatic fabric defect detection method using PRANnet," Applied Sciences, vol. 10, no. 23, p. 8434, 2020.

[84] Y. Li, D. Zhang, and D.-J. Lee, "Automatic fabric defect detection with a wide-and-compact network," Neurocomputing, vol. 329, pp. 329-338, 2019. 
[85] H. Zhou, B. Jang, Y. Chen et al., "Exploring faster RCNN for fabric defect detection," in Proceedings of the 2020 Third International Conference on Artificial Intelligence for Industries (AI4I), Irvine, CA, USA, September 2020.

[86] Z. Zhao, K. Gui, and P. Wang, "Fabric defect detection based on cascade faster R-CNN," in Proceedings of the 4th International Conference on Computer Science and Application Engineering, Sanya, China, October 2020.

[87] J. Wu, J. Le, Z. Xiao et al., "Automatic fabric defect detection using a wide-and-light network," Applied Intelligence, pp. 1-17, 2021.

[88] X. Jun, J. Wang, J. Zhou et al., "Fabric defect detection based on a deep convolutional neural network using a two-stage strategy," Textile Research Journal, vol. 91, no. 1-2, pp. 130-142, 2020.

[89] J. Jing, Z. Wang, M. Rtsch et al., "Mobile-Unet: an efficient convolutional neural network for fabric defect detection," Textile Research Journal, 2020.

[90] H. Zhang, L. Zhang, P. Li et al., "Yarn-dyed fabric defect detection with YOLOV2 based on deep convolution neural networks," in Proceedings of the 2018 IEEE 7th Data Driven Control and Learning Systems Conference (DDCLS), pp. 170-174, Enshi, China, May 2018.

[91] Y.-J. Han and H.-J. Yu, "Fabric defect detection system using stacked convolutional denoising auto-encoders trained with synthetic defect data," Applied Sciences, vol. 10, no. 7, p. 2511, 2020.

[92] X. He, L. Wu, and F. Song, "Research on Fabric defect detection based on deep fusion DenseNet-SSD network," in Proceedings of the IcWCSN 2020: 2020 International Conference on Wireless Communication and Sensor Networks, et al. , Warsaw Poland, May 2020.

[93] K. M. C. Mohammed, "Defective texture classification using optimized neural network structure," Pattern Recognition Letters, vol. 135, pp. 228-236, 2020.

[94] S. Mei, Y. Wang, and G. Wen, "Automatic fabric defect detection with a multi-scale convolutional denoising autoencoder network model," Sensors (Basel, Switzerland), vol. 18, no. 4, 2018.

[95] H. Xie and Z. Wu, "A robust fabric defect detection method based on improved RefineDet," Sensors, vol. 20, no. 15, p. $4260,2020$.

[96] Y. Huang, J. Jing, and Z. Wang, "Fabric defect segmentation method based on deep learning," IEEE Transactions on Instrumentation and Measurement, vol. 70, 2021.

[97] I. J. Goodfellow, J. Pouget-Abadie, M. Mirza et al., "Generative adversarial nets,"vol. 2, pp. 2672-2680, in Proceedings of the 27th International Conference on Neural Information Processing Systems, vol. 2, pp. 2672-2680, MIT Press, Montreal, Canada, December 2014.

[98] K. Yan, A. Chong, and Y. Mo, "Generative adversarial network for fault detection diagnosis of chillers," Building and Environment, vol. 172, p. 106698, 2020.

[99] J. Liu, C. Wang, H. Su et al., "Multistage GAN for fabric defect detection," IEEE Transactions on Image Processing, vol. 29, pp. 3388-3400, 2020.

[100] X. Le, J. Mei, H. Zhang et al., "A learning-based approach for surface defect detection using small image datasets," Neurocomputing, vol. 408, pp. 112-120, 2020.

[101] Y. Zhao, K. Hao, H. He et al., "A visual long-short-term memory based integrated CNN model for fabric defect image classification," Neurocomputing, vol. 380, pp. 259-270, 2020.

[102] J. Wang, Z. Liu, C. Li et al., "Self-attention deep saliency network for fabric defect detection," Communications in
Computer and Information Science, Springer, vol. 1160Singapore, , 2020.

[103] J. Wang, C. Li, Z. Liu et al., Combing Deep and Handcrafted Features for NTV-NRPCA Based Fabric Defect Detection, Springer, Cham, Switzerland, 2019.

[104] J. Lu, J. Wang, D. Chen et al., "A service-oriented tool-chain for model-based systems engineering of aero-engines," IEEE Access, vol. 6, pp. 50443-50458, 2018.

[105] J. Lu, G. Wang, and M. Törngren, "Design ontology in a case study for cosimulation in a model-based systems engineering tool-chain," IEEE Systems Journal, vol. 14, no. 1, pp. 1297-1308, 2020.

[106] X. Zhou, W. Liang, I. K. Wang et al., "Deep learning enhanced human activity recognition for Internet of healthcare Things," IEEE Internet of Things Journal, vol. 7, no. 7, pp. 6429-6438, 2020.

[107] J. Lu, D. Chen, G. Wang, D. Kiritsis, M. Tórngren et al., "Model-based systems engineering tool-chain for automated parameter value selection," in IEEE Transactions on Systems, Man, and Cybernetics: Systems, 2020.

[108] V. Özdemir, "The big picture on the "AI turn" for digital health: the Internet of Things and cyber-physical systems," Omics: A Journal of Integrative Biology, vol. 23, no. 6, pp. 308-311, 2019.

[109] X. Zhou, W. Liang, S. Shimizu, J. Ma, and Q. Jin, "Siamese neural network based few-shot learning for anomaly detection in industrial cyber-physical systems," in IEEE Transactions on Industrial Informatics, vol. 17, no. 8, pp. 5790-5798, 2020.

[110] K. Hanbay, M. F. Talu, and Ö. F. Özgüven, "Fabric defect detection systems and methods-a systematic literature review," Optik, vol. 127, no. 24, pp. 11960-11973, 2016.

[111] K. Yildiz, A. Buldu, M. Demetgul et al., "A novel thermalbased fabric defect detection technique," Journal of the Textile Institute, vol. 106, no. 3, 2015.

[112] B. Fang, X. Long, Y. Zhang et al., "Fabric defect detection using vision-based tactile sensor," 2020, https://arxiv.org/ abs/2003.00839.

[113] S. Bangare, N. Dhawas, V. Taware et al., "Fabric fault detection using image processing," IJARCCE, vol. 6, pp. 405409, 2017.

[114] X. Zhou, W. Liang, K. I.-K. Wang et al., "Academic influence aware and multidimensional network analysis for research collaboration navigation based on scholarly big data," IEEE Transactions on Emerging Topics in Computing, vol. 9, no. 1, pp. 246-257, 2021.

[115] Z. Wang and J. Jing, "Pixel-wise fabric defect detection by CNNs without labeled training data," IEEE Access, vol. 8, pp. 161317-161325, 2020.

[116] L. Chen, N. Yan, H. Yang et al., "A data augmentation method for deep learning based on multi-degree of freedom (DOF) automatic image acquisition," Applied Sciences, vol. 10 , no. 21 , p. $7755,2020$.

[117] W. Wei, D. Deng, L. Zeng et al., "Real-time implementation of fabric defect detection based on variational automatic encoder with structure similarity," Journal of Real-Time Image Processing, pp. 1-17, 2020.

[118] D. Siegmund, T. Samartzidis, B. Fu et al., "Fiber defect detection of inhomogeneous voluminous textiles," in Proceedings of the MCPR 2017, pp. 278-287, Huatulco, Mexico, June 2017.

[119] T. Shunji, N. Kazuki, U. Hideyuki et al., "Research into development of the defect detection system for knitted fabric produced by the circular knitting machines by image 
analysis," Journal of Textile Engineering, vol. 64, no. 2, pp. $45-49,2018$.

[120] R. Schmitt, T. Fürtjes, B. Abbas et al., "Real-time machinevision-system for an automated quality monitoring in mass production of multiaxial non-crimp fabrics," IFAC-PapersOnLine, vol. 10267, no. 3, pp. 769-782.

[121] N.-T. Huynh, "Online defect prognostic model for textile manufacturing," Resources, Conservation and Recycling, vol. 161, p. 104910, 2020.

[122] I. Ilhan, "Concept of industry 4.0 in textile manufacturing processes," Pamukkale University Journal of Engineering Sciences, vol. 25, pp. 810-823, 2019.

[123] K. Yan, W. Shen, Q. Jin et al., "Emerging privacy issues and solutions in cyber-enabled sharing services: from multiple perspectives," IEEE Access, vol. 7, pp. 26031-26059, 2019.

[124] X. Zhou, Y. Hu, W. Liang et al., "Variational LSTM enhanced anomaly detection for industrial big data," IEEE Transactions on Industrial Informatics, vol. 17, no. 5, pp. 3469-3477, 2020.

[125] X. Zhou, X. Xu, W. Liang et al., "Intelligent small object detection based on digital twinning for smart manufacturing in industrial CPS," IEEE Transactions on Industrial Informatics, p. 1, 2021. 